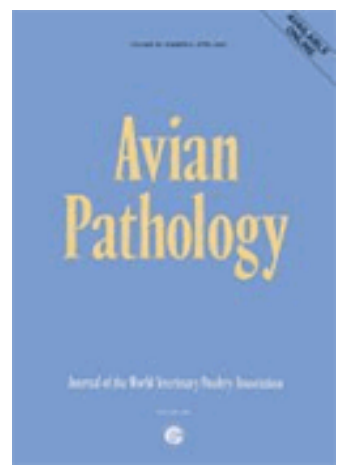

\title{
A field study on the significance of vaccination against infectious bursal disease virus (IBDV) at the optimal time point in broiler flocks positive for maternally derived IBDV antibodies
}

\begin{tabular}{|c|c|}
\hline Journal: & Avian Pathology \\
\hline Manuscript ID: & CAVP-2007-0072.R1 \\
\hline Manuscript Type: & Original Research Paper \\
\hline $\begin{array}{l}\text { Date Submitted by the } \\
\text { Author: }\end{array}$ & 04-Jul-2007 \\
\hline Complete List of Authors: & $\begin{array}{l}\text { Block, Hermann; Poultry Practice Meyer-Block } \\
\text { Meyer Block, Karen; Poultry Practice Meyer-Block } \\
\text { Rebeski, Dierk; Lohmann Animal Health } \\
\text { Scharr, Heike; Lohmann Animal Health } \\
\text { de Wit, Sjaak; GD Deventer } \\
\text { Rohn, Karl; University of Veterinary Medicine Hannover, Institute } \\
\text { for Biometry, Epidemiology and Information Processing } \\
\text { Rautenschlein, Silke; University of Veterinary Medicine Hannover, } \\
\text { Clinic for Poultry }\end{array}$ \\
\hline Keywords: & $\begin{array}{l}\text { infectious bursal disease, broiler, maternal antibodies, field } \\
\text { conditions }\end{array}$ \\
\hline
\end{tabular}

\section{SCHOLARONE Manuscripts}




\section{A field study on the significance of vaccination against infectious bursal disease virus (IBDV) at the optimal time point in broiler flocks with maternally derived IBDV antibodies}

Hermann Block, ${ }^{1}$ Karen Meyer-Block, ${ }^{1}$ Dierk E. Rebeski, ${ }^{2}$ Heike Scharr, ${ }^{2}$ Sjaak de Wit, ${ }^{3}$ Karl Rohn ${ }^{4}$, and Silke Rautenschlein ${ }^{5}$

${ }^{1}$ Poultry Practice Meyer-Block, Uelsen, Germany; ${ }^{2}$ Lohmann Animal Health, Cuxhaven, Germany; ${ }^{3}$ GD Deventer, The Netherlands; ${ }^{4}$ Institute for Biometry, Epidemiology and Information Processing; University of Veterinary Medicine, ${ }^{5}$ Clinic for Poultry, University of Veterinary Medicine Hannover, Germany

Short title: IBDV vaccination under field conditions

*Corresponding Author:

Silke Rautenschlein

Address: $\quad$ Clinic for Poultry ,University of Veterinary Medicine Hannover, Bünteweg 17, 30559 Hannover, Germany

Tel.: $\quad 49-511-953-8763$

Fax: $\quad 49-511-953-8580$

Email: $\quad$ Silke.Rautenschlein@tiho-hannover.de

Received: 27 April 2007

Note to publisher:

There are also alterations needed to the Figures, which I cannot manage!

In Figure 1 - titer needs changing to titre and nummer to number

Titer- titre also needed on Figure 2 


\section{A field study on the significance of vaccination against infectious bursal disease virus (IBDV) at the optimal time point in broiler flocks with maternally derived IBDV antibodies}

Hermann Block, ${ }^{1}$ Karen Meyer-Block, ${ }^{1}$ Dierk E. Rebeski, ${ }^{2}$ Heike Scharr, ${ }^{2}$ Sjaak de Wit, ${ }^{3}$ Karl Rohn ${ }^{4}$, and Silke Rautenschlein ${ }^{5} *$

\section{Abstract}

The right strategy for infectious bursal disease (IBD) control and its success rate under field conditions depends on hygiene management, IBD field pressure, level and variation in maternally derived IBD antibody (MDA) levels, and the IBD-vaccine strains to be used. Usually, standard vaccination programmes are used, which are not always adapted to the specific conditions on the farm and to the strain of chicken. Employing the 'Deventer formula' may help to estimate the optimal time for vaccination for a specific flock based on the MDA level, its variation, the genetic background of the chicken, and the IBD vaccine strain. Two field studies with 16 or 20 commercial broiler flocks were conducted, applying an intermediate IBD vaccine before, at the best, and after the estimated optimal vaccination time estimated by the 'Deventer formula'. These studies showed that flocks which had been IBD-vaccinated between one day before, at, or up to three days after the estimated optimal time point, developed detectable humoral immunity up to 14 days post vaccination. If birds had been vaccinated more than one day before the calculated optimal vaccination date, the humoral immune response was delayed or non-detectable until slaughter. The induction of humoral immunity correlated with the incidence of bursa lesions and IBDV-detection by RT-PCR. As indicated in this study, under field conditions bursa lesions may develop later than predicted based on experimental experiences. The late 
incidence of bursa lesions after vaccination may be confused with field virus-induced lesions, in which case sequencing may offer a valuable tool for differentiation. 


\section{Introduction}

Immunosuppressive diseases are of constant concern for poultry producers worldwide. Infectious bursal disease virus (IBDV) is one of the most important immunosuppressive agents in modern poultry production. Depending on the virulence of the IBDV strain, age at the time of infection, presence of IBDV antibodies and the genetic background of the infected chicken, infection with IBDV may induce a temporary or permanent destruction of the bursa cloacalis and other lymphoid tissues (Lukert \& Saif, 2003). Destruction of B cells and macrophages, and their functions contribute to IBDV-induced immunosuppression (Sharma et al., 2000; Khatri et al., 2005).

Besides hygiene strategies, the current infectious bursal disease (IBD) control methods involve passive and active immunisation (Fussell, 1998). It has been shown that the timing of IBD vaccine administration in broiler progeny is pivotal (Ather, 1993; van den Berg et al., 2000). The optimal vaccination time depends upon the maternally derived antibody (MDA) level of the broiler chicks, the vaccine strain to be used, its breakthrough titre, and the field pressure (de Wit, 1998, 2001, de Wit \& van Loon, 1998). A high variation in MDA levels between birds can make it advisable to vaccinate a broiler flock twice to induce homogeneous protection in birds (McIlroy et al., 1992).

Vaccination in the presence of IBDV antibody levels above the breakthrough titre of the vaccine will lead to a significant delay of IBDV replication and the induction of immunity, as shown by recent laboratory investigations (McCarty et al., 2005; Rautenschlein et al., 2005). Other experimental studies have shown that IBD vaccine virus may even be completely neutralized by maternally derived antibodies 
(van den Berg \& Meulemans, 1991; Tsukamoto et al., 1995; Alam et al., 2002; HairBejo et al., 2004; Moraes et al., 2005). In order to have chickens protected against IBDV field challenge, it is crucial to determine the optimal timing for IBD vaccine delivery (Tsukamoto et al., 1995). The optimal timing is often predicted based on serological data following detection of IBDV MDA by an ELISA system during the first week post hatch (Kouwenhoven \& van den Bos, 1992; 1994). The "Deventer formula" was developed to estimate the optimal vaccination time point based on the half life time of the MDA, the age of the chicken at sampling, genetic background, breakthrough titre of the vaccine, and the requested percentage of the flock having antibody levels below the breakthrough titre of the vaccine at the time of administration (de Wit, 1998, 2001). So far, hardly any studies have been available comparing the outcome of IBDV vaccination at the optimal, before the optimal and after the optimal time point under field conditions in commercial broiler flocks. One recent study indicates that the estimation of the optimal vaccination time in the field, which was based on ELISA antibody titres, may lead to too early vaccination (de Herdt et al., 2005). The technical performance of ELISA systems may vary between manufacturers and laboratories and may affect the interpretation of the maternal antibody levels (de Wit, 2001; de Wit et al., 2007).

Two field studies including 16 and 20 broiler flocks were conducted comparing the IBDV vaccine response in broiler flocks with variable MDA levels that were vaccinated before, at, or after the estimated optimal time point for a single IBDV vaccination. Besides the induction of humoral immunity, we also looked at the development of IBDV-induced bursa lesions and detected and characterized the IBDV genome in bursa samples of vaccinated flocks. The overall performance of the flock was evaluated. To our knowledge this is the first study comparing the IBDV vaccine 
response in broilers with different levels of maternally derived antibodies under field conditions.

\section{Materials and Methods}

Chickens. Overall, 36 commercial broiler flocks with 20,000 to 140,000 chickens per farm (14,000-48,000 broilers/house) in the North-Western region of Lower Saxony in Germany participated in the two field trials. These are representative farms for this region, which had already established a vaccination regime against IBDV and were under regular veterinary control. Broilers were raised under controlled conditions based on national animal welfare regulations. The broilers belonged to the hybrid lines Cobb 500, Ross 308 and Ross 508. Besides vaccination against IBDV, birds were also vaccinated by standard procedures against infectious bronchitis by spray at day of hatch and Newcastle disease at 7 days by drinking water. The broiler breeder flocks had been vaccinated against IBDV with an intermediate live vaccine; some breeders had also been boosted with an inactivated IBDV vaccine between 16 and 17 weeks of age (see Table 2 for data on Trial 1; in Trial 2 only flock 18 had been boosted with an inactivated IBDV vaccine).

Vaccine. A commercially available intermediate IBDV vaccine strain was used. As previous laboratory studies indicated, this vaccine induces bursa lesions around 3 days post inoculation in specific pathogen free (SPF) layer-type and antibody free broiler chickens (Jung, 2007). Recovery from bursa lesions would be expected to take place around 10 days post vaccination of antibody free birds (Jung, 2007). One dose consisted of at least $10^{3}$ median egg-infectious doses $\left(\mathrm{EID}_{50}\right)$ per bird. Vaccination 
was performed based on the manufacturer's recommendations by drinking water. The break through titre of this intermediate vaccine in the IDEXX enzyme linked immunosorbent assay (ELISA) is 1: 125. This ELISA titre corresponds to the neutralizing maternal antibody levels at the optimal time of vaccination (Lucio \& Hitchner, 1979; Skeeles et al., 1979; Lukert \& Saif, 2003). Because the IDEXX ELISA is very commonly used in the field, in the present study, we refer to the break through IDEXX ELISA titre.

Serology. IBDV antibodies were detected in collected serum samples using a commercially available ELISA kit (FlockChek® IBD ELISA, IDEXX, Germany) and titres were calculated as described by the manufacturer.

Deventer formula. The optimal vaccination time was determined by the Deventer formula (de Wit, 1998; 2001):

Vaccination age $=\left\{\left(\log _{2}\right.\right.$ IBDV antibody ELISA titre of the bird $(\%)-\log _{2}$ breakthrough titre of the vaccine) $\left.\times t^{1 / 2}\right\}+$ age at sampling + correcting value $0-4$ In which

Bird (\%): $\quad$ titre of the bird (at sampling) that represents a certain percentage of the flock (in this study: $75 \%$ ) that is desired to be susceptible to the vaccine at the time of the application Breakthrough: breakthrough (ELISA) titre of the vaccine to be used (for this vaccine in the ELISA system used, a titre of 125)

$\mathrm{t} 1 / 2$ : half life time of the antibodies (ELISA titre) in the type of chickens that were sampled (broilers: 3 days)

Age at sampling: age of the birds at sampling 
Correcting value 0-4: extra days when the sampling was done at 0-4 days post hatch.

Histology. Bursae cloacalis were collected post mortem and fixed in 10\% phosphate buffered formalin, paraffin embedded, cut and stained with haematoxylin and eosin. Bursa lesion scores were determined microscopically and compared between groups (Muskett et al., 1979). The scoring system was as follows: score 0: no lesions; score 1: 1-25\% of follicles show lymphoid depletion (less then $50 \%$ depletion per follicle), accumulation of heterophils; score 2: 26-50 \% of follicles show almost complete lymphoid cell depletion (more than $75 \%$ depletion per follicle), necrosis and accumulation of heterophils; score 3: 51-75\% of follicles show almost complete depletion of bursa follicles with necrosis and heterophils; score 4: 76-100\% of follicles show almost complete depletion of bursa follicles with necrosis and heterophils, hyperplasia and cysts may be observed; score 5: $100 \%$ of follicles show almost complete depletion of bursa follicles with loss of bursa architecture, and fibrosis.

Detection of IBDV by RT-PCR and sequencing of the VP2-region. Five bursal samples per flock were pooled for the detection of IBDV by RT-PCR. Total RNA was isolated using the TriPure Isolation Reagent (Roche) following the guidelines of the manufacturer. RNA isolation was followed by a RT-PCR reaction with the RNAAmplification Kit SYBR Green I (Roche). The following primers were used: forward: 5'-GGT AGC CAC ATC TGA CAG-3' (Boot et al., 1999); reverse 5'-CGC TCG AAG TTR CTC ACC C-3' (Islam et al., 2001). The RT-PCR was performed at the following conditions: RT-reaction for $30 \mathrm{~min}$ at $52^{\circ} \mathrm{C}$; denaturation for $30 \mathrm{sec}$ at $95^{\circ} \mathrm{C}$, 
40 cycles with $5 \mathrm{sec}$ at $95^{\circ} \mathrm{C}, 10 \mathrm{sec}$ at $57^{\circ} \mathrm{C}$, and $30 \mathrm{sec}$ at $72^{\circ} \mathrm{C}$. The RT-PCR was followed by a melting curve analysis. Sequencing of the RT-PCR product of $540 \mathrm{bp}$ was conducted with the described forward and reverse primer at BaseClear (Leiden, NL).

Reisolation of IBDV. Pools of bursal homogenate were inoculated into 10-day-old SPF embryonated chicken eggs following standard procedures (Rosenberger et al., 1998). At 5 days post inoculation, the allantoic fluid was harvested and RT-PCR was performed as described above to detect the IBDV genome.

Production parameters. The following parameters were collected and evaluated to compare the production values between flocks:

Total animal loss $\left(\right.$ loss $\left._{\text {total }}\right)=$ animal loss $(\%)$ during the production period + condemnation at slaughter $(\%)$.

$D W G$ (daily weight gain $)=$ final weight at slaughter $(\mathrm{g}) /$ fattening days. $F C R($ feed conversion rate) $=$ feed conversion $/$ final total live weight

Production index $=\left\{100-\operatorname{loss}_{\text {total }}(\%)\right\} \times$ DWG $/$ FCR x 10

Experimental protocol. Trial 1: Sixteen broiler farms were selected as described. Serum samples were collected from 30 randomly chosen birds/flock between 3 and 12 days of age, and at a second time between 2 to 5 days before vaccination, to detect maternal IBDV antibodies, to estimate and confirm the optimal time for vaccination by the Deventer formula (Table 1). At the estimated optimal time, birds were vaccinated by drinking water with one dose of the intermediate IBDV vaccine 
following the manufacturer's instructions. At day of vaccination, and in weekly intervals until slaughter, all flocks were observed for clinical disease, mortality and performance. At the same times except the day of vaccination, 30 serum samples were collected randomly for IBDV antibody detection, and five randomly chosen broilers were killed to determine bursal lesions. Bursal samples were taken for IBDV detection by RT-PCR and histology. During the fattening period all dead birds were examined post mortem to determine the cause of death.

Trial 2: Twenty broiler flocks were selected as described above. Unlike in Trial 1, in Trial 2 broiler flocks were vaccinated based on the recommendation of the breeder companies or the hatcheries. Serum samples $(n=24 /$ flock $)$ that had been taken between the $3^{\text {rd }}$ and $7^{\text {th }}$ day post hatch were used for the retrospective estimation of the optimal IBD vaccination time using the Deventer Formula. Based on the difference between the actual time of vaccination and the retrospectively estimated optimal time, the broiler flocks were divided into three groups: birds vaccinated 8 to 1 day before the estimated optimal time for vaccination (group 1); birds vaccinated at the optimal time (group 2); and birds vaccinated up to 6 days after the optimal time (group 3; Table 3). Vaccination was conducted as described in Trial 1. Further serum samples $(n=30)$ were collected at the day of vaccination, and in weekly intervals until slaughter. Clinical observations and production parameters were obtained weekly. Mortality rates were determined and all dead birds were examined to identify the cause of death.

Statistical analysis. All collected data were included in a descriptive analysis. The log-normal distribution of model residuals of IBDV antibody titres was confirmed by visual assessment of normal probability plots and by the Shapiro-Wilk Test. For these 
parameters logarithm to the base 2 transformation was performed prior to analysis; the description is shown as box-plots of original data. Bursal lesion scores as ordinal scaled data are neither normally nor log-normally distributed, because of that nonparametric methods were applied.

Differences in IBDV antibody development between flocks depending on vaccination before, at, and after the optimal time point were compared. Two-way analysis of variance was used with the flock as an independent effect and time-points of vaccination between flocks as repeated measurements with Tukey's post-hoc test for multiple pair wise comparisons. Significance was defined as $P \leq 0.05$.

Analyses were carried out with the statistical software SAS, version 9.1 (SAS Institute, Cary, NC). For the analysis of the linear model, the procedure GLM (General Linear Model) was used. As nonparametric methods we used Kruskal-Wallis and Wilcoxon two sample test for independent samples.

\section{Results}

Estimation of the optimal vaccination time. In field Trial 1, 30 serum samples/flock were investigated for IBDV MDA by ELISA within the first two weeks post hatch. Based on the Deventer formula the optimal vaccination time for these broiler flocks in this trial was between 17 and 23 days post hatch (Table 1). IBDV antibody titres in 30 serum samples/flock taken at 2-5 days before the estimated optimal vaccination time confirmed the estimated optimal vaccination time (Table 2). All flocks showed the expected drop in MDA levels (Table 2). 
In Trial 2, all broiler flocks were vaccinated based on the recommendation of the breeder companies or the hatcheries. Serum samples were taken between the $3^{\text {rd }}$ and $7^{\text {th }}$ day post hatch for the retrospective estimation of the optimal IBD vaccination time. Based on the difference between the actual time of vaccination and the retrospectively estimated optimal time, the broiler flocks were divided into three groups (Table 3): four flocks that had been vaccinated between 8 to 1 day before the estimated optimal time for vaccination (group 1); five flocks that had been vaccinated at the estimated optimal time (group 2); and 11 flocks that had been vaccinated between one and six days after the optimal time (group 3). Antibody detection in Trial 2 at the day of vaccination revealed that birds in group 1 had a significantly higher antibody level (titre range 25-6590) than birds of groups 2 (titre range 6-3126) or 3 (titre range 2-2680) (data not shown; $P<0.05$ ). In flock 9, which was vaccinated eight days before the optimal vaccination time, $92 \%$ of the serum samples had IBDV antibody levels above the estimated cut-off value of 125 in the ELISA system used (Table 3). With the exception of flock 1, all the other flocks in group 2 had MDA levels above the cut-off value in less than $25 \%$ of the tested birds/flock (Table 3 ). In group 3, which was vaccinated after the optimal vaccination time, at the time of vaccination $0-17 \%$ of the tested birds had MDA levels above the cut-off value. Fortyfive percent of the tested flocks of group 3 had only birds with antibody levels below the cut-off value (Table 3).

Induction of humoral immunity after IBDV-vaccination. In Trial 1, all broiler flocks showed seroconversion before slaughter (Figures 1a, 1b and 1c). Only one flock (flock 13) showed a significant increase in IBDV ELISA antibodies at 7 days post vaccination (DPV; Figure 1a) in comparison to antibody levels detected 4-7 days 
before vaccination ( $P<0.05$; data not shown). At 14 DPV, flocks 1, 4, 6, 7, 10, and 14 had comparable antibody levels to their IBDV antibody titres at 7 DPV (Figure 1a,b). $(P>0.05)$. Flocks 8,12 and 16 had significantly higher antibody levels at 14 DPV in comparison to flocks $2,3,5,9,11,14$ and $15(P<0.05)$. With the exception of flock 13, all other flocks showed a significant increase in IBDV antibody levels at $21 \mathrm{DPV}$ in comparison to titres at $7 \mathrm{DPV}(P<0.05)$.

In Trial 2, only three flocks of group 3 (flocks 14, 17, 20, vaccinated at 5 or 6 days after the estimated optimal time) showed a significant increase in IBDV antibodies at $7 \mathrm{DPV}$ in comparison to the day of vaccination $(P<0.05)$, while no significant seroconversion was detected in any of the other 17 flocks at this time (Figure 2a,b). At 14 DPV, all flocks of groups 2 and 3 showed a significant increase in IBDV antibodies in comparison to antibody levels of the same flock at day of vaccination (Figure 2a,c). In group 1 only birds that had been vaccinated 1 day before the optimal vaccination time showed a significant increase in IBDV antibody production at $14 \mathrm{DPV}$ in comparison to $7 \mathrm{DPV}$ (Figure 2b,c; $P<0.05$ ). At $21 \mathrm{DPV}$, all flocks except flock 9 (group 1), which had been vaccinated 8 days before the optimal vaccination time point, had seroconverted.

Induction of histological bursa lesions. In Trial 1, five bursae per flock were evaluated histologically for IBDV specific lesions at different times before and after vaccination. None of the investigated flocks showed bursa lesions at the time of vaccination (data not shown). At 7 DPV, 14 of 16 flocks had detectable bursa lesions (Figure 3). In flock 12 and 13 bursa lesion scores were detected in three out of five, and four out of five chickens, respectively. At 14 DPV, all vaccinated flocks had developed lesions with an average bursa lesion score of $<2$ for flocks 4 and 14; 
scores of 2 to 3 for flocks 1, 11, 15, and 16; and scores of 3.1- 4.2 for flocks 2, 3, 5, 6, $7,8,9,10,12$, and 13 .

Detection of IBDV in bursal tissue. In Trial 1, no IBDV was detected by RT-PCR in bursal tissue at the day of vaccination (data not shown). As indicated in Figure 3, some of the flocks already had IBDV positive bursal samples at 7 DPV. By 14 DPV, all flocks were confirmed as IBDV positive. In flock 5, 8, and 9, IBDV was only detected at the indicated times by RT-PCR after previous propagation of the virus in chicken embryos. By 21 DPV 58\% of the investigated flocks were still positive for IBDV by RT-PCR (Figure 3). Sequencing of the VP2-region of IBDV, which had been amplified from bursal samples of the investigated flocks by RT-PCR, revealed that the IBDV strain detected was solely the administered IBDV vaccine strain (data not shown). The sequence homology between the predicted amino-acid sequences of the RT-PCR products and the original vaccine strain was $100 \%$. After propagation of the bursal samples in embryonated eggs (samples of flocks 5, 8, 9) and subsequent sequencing of the RT-PCR products we detected a sequence homology to the original vaccine stain of 99.7\%. Amino-acid variations were detectable at position 316 (Arg $\rightarrow$ Lys) and 325 (Ile $\rightarrow$ Met).

\section{Health status, post-mortem results and production parameters of the broiler}

flocks. None of the 16 broiler flocks of Trial 1 showed any clinical disease during the growing period. Total animal loss ranged between 2.2 and $6.2 \%$ in the different flocks. Post mortem examination of the dead birds and the randomly selected five birds per flock, which had been killed before and at 7, 14, and 21 days post vaccination, revealed sporadic diagnosis of pericarditis (flock 1 and 7, in one of five 
birds each), or polyserositis (flock 11, one of five birds). Mild catarrhal enteritis was detected in one of five broilers of each of the following flocks: 1, 2, 4, 9, and 15.

Eimeria acervulina was detected in the gut of two of five birds each of flocks 2, 9, and 13 between the $25^{\text {th }}$ and $29^{\text {th }}$ days post hatch. No IBDV-specific macroscopical bursa lesions such as gelatination, haemorrhages or necrosis were seen.

The production indexes varied between 286 and 352 (Trial 1). On average the fattening period lasted 40 days with a daily weight gain between 51.88 and 61.95 g/day. The feed conversion rate ranged between 1.608-1.766.

In Trial 2, none of the 24 broiler flocks showed any clinical disease during the growing period. The total animal loss ranged between 1.9 and $5.6 \%$ in the different flocks. Examination of the dead birds and the randomly selected five birds per flock at the different times during the field trial revealed sporadic diagnosis of lesions in the respiratory or digestive tract. At 7 or $14 \mathrm{DPV}$, one or two of five investigated birds of flocks $1,7,9,11,14,15$, and 19 showed mild catarrhal enteritis. Aerosacculitis was detected in two of five birds of flocks 2 and 12 at 14 DPV. E. acervulina was detected in the gut of birds from flocks 20 and 11 at 14 or 21 DPV, respectively. No IBDVspecific macroscopical bursa lesions such as gelatination, haemorrhages or necrosis were seen.

The production indexes were between 267 and 326 (Trial 2). On average the fattening period lasted 41 days with a daily weight gain between 49.2 and $57.9 \mathrm{~g} /$ day. The feed conversion rate ranged between 1.609 and 1.859 .

\section{Discussion}

This is the first study to demonstrate the importance of keeping to the optimal 
time for IBDV vaccination under field conditions in order to achieve a detectable immune response. Previous observations in the field and experimental studies under laboratory conditions had indicated that high MDA at the time of IBDV vaccination may interfere with the vaccine response and neutralise the vaccine virus (van den Berg \& Meulemans, 1991; Tsukamoto et al., 1995; van den Berg, 2000; Alam et al., 2002; Hair-Bejo et al., 2004; Moraes et al., 2005). But no information has been available so far from controlled field conditions about the real influence of MDA on the outcome of the IBDV vaccine response, accompanied by consecutive monitoring of the flocks. In two large field trials we determined the effect of MDA on the vaccine response of broilers to one intermediate IBDV strain. In the first trial the vaccine response was investigated in broiler flocks vaccinated at the optimal vaccination time estimated with the Deventer formula (de Wit, 1998; 2001). All vaccinated flocks of Trial 1 seroconverted within 14 days of IBDV vaccination. IBDV antibody development correlated with the incidence of IBDV-induced bursal lesions and IBDV detection by RT-PCR. These observations were confirmed in the second trial. Furthermore, the vaccine response of optimally vaccinated birds was compared in the second trial with the vaccine response of birds which had been vaccinated earlier or later than the optimal vaccination time. Our study clearly confirms previous laboratory investigations that high levels of MDA interfere with a homogeneous IBDV vaccine response, and delay or even prevent the induction of humoral immunity (van den Berg \& Meulemans, 1991; Goddard et al., 1994; Tsukamoto et al., 1995; Alam et al., 2002; Hair-Bejo et al., 2004; Jung, 2006). A delayed or prevented immune response may subsequently lead to broilers being susceptible to IBDV field challenge (van den Berg et al., 1991; Kouwenhoven \& van den Bos, 1992; de Wit \& van Loon, 1998). It is not clear from this study what would be the effect on the 
protective immunity of the immunized flock of vaccination at different days ' too early' compared to the optimal calculated time. Consecutive challenge studies of broilers from each flock at different days post vaccination would have been necessary to provide information regarding this point. But certainly our study shows that the Deventer formula provides a useful tool to estimate the optimal vaccination time in order to induce detectable humoral immunity in a timely manner. Future studies are needed to determine how flexible the optimal vaccination day will be under field conditions. In our study it was shown that the IBDV antibody levels at 21 DPV were comparable between chickens vaccinated at the optimal, and one day before the optimal, time. Further investigated is necessary to determine the effect of 'too early' vaccination on the outcome of the vaccine response.

The different genetic backgrounds of the broiler flocks (Ross and Cobb) seem not to have had an influence on the outcome of the IBDV vaccine response (data not shown). Previous laboratory studies have confirmed that the genetic background of the chicken may not influence the pathogenesis of an intermediate IBDV strain (Jung, 2006), whereas the immune responses to virulent strains may vary significantly between chicken lines (Ruby et al., 2006). Furthermore, the vaccination scheme of the parent flocks under field conditions did not significantly influence the outcome of the vaccine response of the progeny as seen in Trial 1 . Based on the variation in MDA levels between progeny of parents which had been vaccinated only with one dose of an IBDV live strain, we may speculate that some of the parent flocks may have gone through a field challenge resulting in unexpected high levels of circulating antibodies. These antibody levels were comparable to titres of parents which had been boosted with an inactivated IBDV vaccine (Table 2). The heterogenicity of the MDA levels in 
the progeny broiler flocks varied between flocks but the levels where still homogeneous enough to justify only a single vaccination (de Wit, 2001).

As demonstrated in field trial 1 , the induction of humoral immunity clearly correlated with the induction of bursal lesions and IBDV replication, as shown previously under experimental conditions (Rautenschlein et al., 2005). If birds were vaccinated at the optimal time, all vaccinated flocks developed IBDV antibodies as well as bursal lesions up to 14 DPV. These studies show that bursal lesions may develop later than would be expected from studies in SPF layer-type chickens (Tanimura \& Sharma, 1998; Kim et al., 1999). This is possibly due to residual levels of MDA (McCarty et al., 2005; Rautenschlein et al., 2005). Furthermore, the bursal lesion scores were unexpectedly high. Most investigated flocks had severe average lesion scores of over 3.3 at one of the investigated times. Interestingly, we did not observe any regeneration of bursal tissue, as would be expected after vaccination with this strain or other intermediate strains, based on laboratory studies (Kim et al., 1999; Alam et al., 2002; Rautenschlein \& Haase, 2005; Jung, 2006). Under field conditions, circulating IBDV vaccine virus may lead to deviating observations in comparison to experimental laboratory studies. Furthermore, slight variation of MDA levels at the time of vaccination may also contribute to an 'extended' period of severe lesions after vaccination on the basis of the whole flock.

The observed high bursal lesion scores in Trial 1, which for many flocks reached its peak between 14 and 21 DPV, may easily be confused with field virusinduced lesions (Ezeokoli et al., 1990; van den Berg et al., 2000). But sequencing of the VP2 region of IBDV, which was detected by RT-PCR in the bursal samples, confirmed solely the detection of the vaccine strain in all 16 flocks. No field virus was detected during these trials. This may be due to low field pressure of virulent IBDV 
strains or to good protection and displacement of the field virus. Experimental studies with this vaccine strain in broilers indicated that it protected vaccinated broilers against challenge with very virulent IBDV (Jung, 2006). Boilers, which had been vaccinated with this intermediate strain were protected against morbidity, mortality and the development of severe bursa lesions (score $>3$ ) in comparison to nonvaccinated broilers, in which $100 \%$ of the birds challenged with very virulent IBDV had developed lesion scores of $>3$ (Jung, 2006).

Despite the high bursa lesion scores and the possibility of at least a temporary immunosuppression due to the IBDV vaccination (Kim et al., 1999; Kim \& Sharma, 2000; Sharma et al., 2000; Rautenschlein et al., 2007), the performance of the broiler flocks was not affected. All flocks had production indices which coincided with field experiences. The observation of pathological lesions in only some of the birds examined post mortem was related to bacterial or parasitic infections but not to IBD, which indicates that some individual birds became sick without affecting the entire flock.

IBD vaccine virus was detected in Trial 1 in $93 \%$ and $58 \%$ of the broiler flocks at 14 and 21 DPV respectively. One flock was positive by RT-PCR only at 7 DPV. Since the sample size was small and the bursae were pooled from each flock for IBDV detection, we may not have detected all positive flocks at the indicated times due to the limitations of the detection method (Smiley et al., 1999). The histological bursa lesions of chickens which had been IBDV-negative by RT-PCR, indicate that virus replication had taken place in all investigated flocks.

Overall, our study demonstrated the importance of the estimation of the optimal vaccination time for IBDV vaccination under field conditions. The Deventer formula was shown to be a useful tool for the estimation of the optimal vaccination 
time. We demonstrated that under field conditions, vaccine induced bursa lesions may be detected later and may be more severe, as would be expected from laboratory investigations with SPF chickens. Previous experimental studies with MDA-positive commercial broilers using other intermediate IBDV strains support this observation (Rautenschlein et al., 2005). It is possible that re-circulating vaccine virus may interfere with the clearance of the vaccine virus from the flock. This may especially be true in flocks with heterogeneous MDA levels that allow infections of the vaccine virus to take place in a staggered manner. These vaccine-induced bursa lesions may easily be confused with field virus challenge. Sequencing of the detected virus, however allows differentiation between field and vaccine strains.

Finally, it needs to be remembered that only one intermediate IBDV vaccine strain was tested in this field studies. Because this vaccine strain may differ in its virulence and other characteristics from other intermediate IBDV vaccine strains, our observations cannot necessarily be extrapolated to other intermediate IBD vaccines.

\section{References}

Alam, J., Rahman, M. M., Sil, B. K., Khan, M. S. R., Giasuddin \& Sarker, M. S. K. (2002). Effect of maternally derived antibody on vaccination against infectious bursal disease (Gumboro) with live vaccine in broiler. International Journal of Poultry Science, 1, 98-101.

Ather, M. A. (1993). Infectious bursal disease in chickens. Poultry Advisor, 26, 27-30.

Boot, H. J., ter Huurne, A. A., Peeters, B. P. \& Gielkens, A. L. (1999). Efficient rescue of infectious bursal disease virus from cloned cDNA: evidence for 
involvement of the 3'-terminal sequence in genome replication. Virology, 265, $330-341$.

de Herdt, P., Jagt, E., Paul, G., Van Colen, S., Renard, R., Destrooper, C. \& van den Bosch, G. (2005). Evaluation of the enzyme-linked immunosorbent assay for the detection of antibodies against infectious bursal disease virus (IBDV) and the estimation of the optimal age for IBDV vaccination in broilers. Avian Pathology, 34, 501-504.

de Wit, J.J \& van Loon A.A. W.M. (namens de Gumboro-werkgroep) (1998).

Gumboro-vaccinatie. Tijdschrift voor Diergeneeskunde, 123, 7-10

de Wit, J.J. (1998). Gumboro disease: estimation of optimal time of vaccination by the Deventer formula. Polish Veterinary Journal, 3, 19-22.

de Wit, J.J. (2001). Gumboro Disease: estimation of optimal time of vaccination by the Deventer formula. Annual report and proceedings of COST Action 839: Immunosuppressive viral diseases in poultry, pp 170-178.

de Wit, J. J., Heijmans, J. F., Mekkes, D. R. \& van Loon, A. A. W. M. (2001). Validation of five commercially available ELISAs for the detection of antibodies against infectious bursal disease virus (serotype 1). Avian Pathology, 30, 543-549.

de Wit, J.J., van de Sande, H.W.A., Counotte, G.H.M. \& Wellenberg, G.J. (2007). Analyses of the results of different test systems in the 2005 global proficiency testing schemes for Infectious Bursal Disease Virus and Newcastle Disease Virus antibody detection in chicken serum. Avian Pathology (accepted).

Ezeokoli, C. D., Ityondo, E. A., Nwannenna, A. I. \& Umoh, J. U. (1990). Immunosuppression and histopathological changes in the bursa of Fabricius 
associated with infectious bursal disease vaccination in chicken. Comparative Immunology, Microbiology and Infectious Diseases, 13, 181-188.

Fussell, L. W. (1998). Poultry industry strategies for control of immunosuppressive diseases. Poultry Science, 77, 1193-1196.

Goddard, R. D., Wyeth, P. J. \& Varney, W. C. (1994). Vaccination of commercial layer chicks against infectious bursal disease with maternally derived antibodies. Veterinary Record, 135, 273-274.

Hair-Bejo, M., Ng, M.K. \& Ng, H.Y. (2004). Day old vaccination against infectious bursal disease in broiler chickens. International Journal of Poultry Science, 3, 124-128.

Islam, M. R., Zierenberg, K., Eterradossi, N., Toquin, D., Rivallan, G. \& Müller, H. (2001). Molecular and antigenic characterization of Bangladeshi isolates of infectious bursal disease virus demonstrate their similarities with recent European, Asian and African very virulent strains. Journal of Veterinary Medicine B Infectious Diseases Veterinary Public Health, 48, 211-221.

Jung, A. (2006). Pathogenesestudie eines intermediärvirulenten Gumborovirus in spezifiziert-pathogen-freien (SPF) Hühnern und kommerziellen Broiler. Doctoral Thesis; University of Veterinary Medicine Hannover.

Khatri, M., Palmquist, J. M., Cha, R. M. \& Sharma, J. M. (2005). Infection and activation of bursal macrophages by virulent infectious bursal disease virus. Virus Research, 113, 44-50.

Kim, I. J., Gagic, M. \& Sharma, J. M. (1999). Recovery of antibody-producing ability and lymphocyte repopulation of bursal follicles in chickens exposed to infectious bursal disease virus. Avian Diseases, 43, 401-413. 
Kim, I. J. \& Sharma, J. M. (2000). IBDV-induced bursal T lymphocytes inhibit mitogenic response of normal splenocytes. Veterinary Immunology and Immunopathology, 74, 47-57.

Kouwenhoven, B. \& van den Bos, J. (1992). Control of very virulent infectious bursal disease (Gumboro disease) in the Netherlands with more virulent vaccines. Proceedings of the World's Poultry Congress. (pp. 465-468). Amsterdam, The Netherlands.

Kouwenhoven, B. \& van den Bos, J. (1994). Control of very virulent infectious bursal disease (Gumboro disease) in the Netherlands with more virulent vaccines. Proceedings of the International Symposium on Infectious Bursal Disease and Chicken Infectious Anemia. (pp. 262-279). Rauischholzhausen, Germany.

Lucio, B. \& Hitchner, S. B. (1979). Infectious bursal disease emulsified vaccine: Effect upon neutralizing antibody levels in the dam and subsequent protection of the progeny. Avian Disease, 23, 466-478.

Lukert, P. D. \& Saif, Y. M. (2003) Infectious bursal disease. In Y. M. Saif, J. R. Glisson, A. M. Fadly, L. R. McDougald, \& D. E. Swayne. (2003). Diseases of Poultry 11 edn (pp. 161-180) Ames: Iowa State University Press.

McCarty, J. E., Brown, T. P. \& Giambrone, J. J. (2005). Delay of infectious bursal disease virus infection by in ovo vaccination of antibody positive chicken eggs. Journal of Applied Poultry Research, 14, 136-140.

McIlroy, S. G., Goodall, D. W., Bruce, D. W., McCracken, R. M. \& McNulty, M. S. (1992). The cost benefit of vaccinating broiler flocks against subclinical infectious bursal disease. Avian Pathology, 21, 65-76.

Moraes, H.L.S., Salle, C.T.P., Nascimento, V.P., Salle, F.O., Rocha, A.C.G.T., Souza, G.F., Furian, T.Q. \& Artencio, J.O. (2005). Infectious bursal disease: 
Evaluation of maternal immunity and protection by vaccination of one-day old chicks against challenge with very virulent virus isolate. Brazilian Journal of Poultry Science, 7, 51-57.

Muskett, J. C., Hopkins, I. G., Edwards, K. R. \& Thornton, D. H. (1979). Comparison of two infectious bursal disease vaccine strains: efficacy and potential hazards in susceptible and maternally immune birds. Veterinary Record, 104, 332-334.

Rautenschlein, S. \& Haase, C. (2005). Differences in the immunopathogenesis of infectious bursal disease virus (IBDV) following in ovo and post-hatch vaccination of chickens. Veterinary Immunology and Immunopathology, 106, 139-150.

Rautenschlein, S., Kraemer, C., Vanmarcke, J. \& Montiel, E. (2005). Protective efficacy of intermediate and intermediate plus infectious bursal disease virus (IBDV) vaccines against very virulent IBDV in commercial broilers. Avian Diseases, 49, 231-237.

Rautenschlein, S., Kraemer, C., Montiel, E., Vanmarcke, J. \& Haase, C. (2007). Bilateral effects of vaccinations against infectious bursal disease and Newcastle disease investigated in specific-pathogen-free layer type and commercial broiler-type chicken. Avian Diseases, 51, 14-20.

Rosenberger, J. K., Saif, Y. M. \& Jackwood, D. J. (1998) Infectious bursal disease. In D. Swayne, Glission, J. R., Jackwood, M. W., Pearson, J. E., Reed, W. M. (1998). Isolation and identification of avian pathogens (pp. 215-218) Kennett Square: American Association of Avian Pathologists.

Ruby, T., Whittaker, C., Withers, D. R., Chelbi-Alix, M. K., Morin, V., Oudin, A., Young, J. R. \& Zoorob, R. (2006). Transcriptional profiling reveals a possible 
role for the timing of the inflammatory response in determining susceptibility to a viral infection. Journal of Virology, 80, 9207-9216.

Sharma, J. M., Kim, I. J., Rautenschlein, S. \& Yeh, H. Y. (2000). Infectious bursal disease virus of chickens: pathogenesis and immunosuppression. Developmental and Comparative Immunology, 24, 223-235.

Skeeles, J. K., Lukert, P. D., Fletcher, O. J. \& Leonard, J. D. (1979). Immunization studies with a cell-culture adapted infectious bursal diseas virus. Avian Diseases, 23, 456-465.

Smiley, J. R., Sommer, S. E. \& Jackwood, D. J. (1999). Development of a ssRNA internal control reagent for an infectious bursal disease virus reverse transcription/polymerase chain reaction-restriction fragment length polymorphism diagnostic assay. Journal of Veterinary Diagnostic Investigations, 11, 497-504.

Tanimura, N. \& Sharma, J. M. (1998). In-situ apoptosis in chickens infected with infectious bursal disease virus. Journal of Comparative Pathology, 118, 15-27.

Tsukamoto, K., Tanimura, N., Kakita, S., Ota, K., Mase, M., Imai, K. \& Hihara, H. (1995). Efficacy of three live vaccines against highly virulent infectious bursal disease virus in chickens with or without maternal antibodies. Avian Diseases, $39,218-229$.

van den Berg, T. P. \& Meulemans, G. (1991). Acute infectious bursal disease in poultry: protection afforded by maternally derived antibodies and interference with live vaccination Avian Pathology, 20, 409-421.

van den Berg, T. P., Gonze, M. \& Meulemans, G. (1991). Acute infectious bursal disease in poultry, isolation and characterisation of a highly virulent strain. Avian Pathology, 20, 133-143. 
van den Berg, T. P., Eterradossi, N., Toquin, D. \& Meulemans, G. (2000). Infectious bursal disease (Gumboro disease). Revue Scientifique Technique, 19, 509-543. 


\section{Figure legends}

Figure 1: IBDV antibody development after vaccination at the optimal time point (Trial 1). Serum samples were collected at $7(a), 14(b)$, and 21 (c) days post vaccination and tested for IBDV antibodies by ELISA. $n=30 / f l o c k$. The ELISA data are presented in the box \& whisker diagram: the short line within the grey rectangular box represents the median of the variables; the upper and lower boarder of the rectangular box represent the 25 and 75 quartiles, respectively; the whiskers indicate highest and lowest value; outliers are indicated by + .

Figure 2: IBDV antibody development after vaccination before (days $-8,-3,-1$ ), at (day 0), and after the optimal time point (days 1 to 6) (Trial 2). Serum samples were collected at the day of vaccination (a), $7(b), 14(c)$, and 21 (d) days post vaccination and tested for IBDV antibodies by ELISA; at 21days post vaccination, flocks vaccinated at day 2 and 6 after the optimal time had been slaughtered so no data are available for these flocks at this time. 30 serum samples/flock; the number of flocks per day varied: day $-8,-1,+1,+5: n=1 ;$ day $-3,+2,+3,+6: n=2 ;$ day $+4: n=3$; day 0: $n=5$. The ELISA data are presented in the box \& whisker diagram: the short line within the grey rectangular box represents the median of the variables; the upper and lower boarder of the rectangular box represent the 25 and 75 quartiles, respectively; the whiskers indicate highest and lowest value; outliers are indicated by $+$. 
Figure 3: Development of histological bursa lesions and detection of IBDV by RTPCR after vaccination of broiler flocks with an intermediate IBDV strain (Trial 1). Flocks 3, 8, 9, and 16 were slaughtered before 21 days post vaccination and no data were available for this time. $d p v=$ days post IBDV vaccination. $n=5$ bursae per flock were investigated. $+=$ detection of IBDV by RT-PCR from pooled bursa tissue $(n=5 / p o o l) ;-=$ no detection of IBDV by RT-PCR. IBDV detection in flocks 5, 8, 9 was possible after propagation of IBDV in embryonated chicken eggs followed by RTPCR. 
Table 1. Estimation of the optimal IBD vaccination time in Trial 1

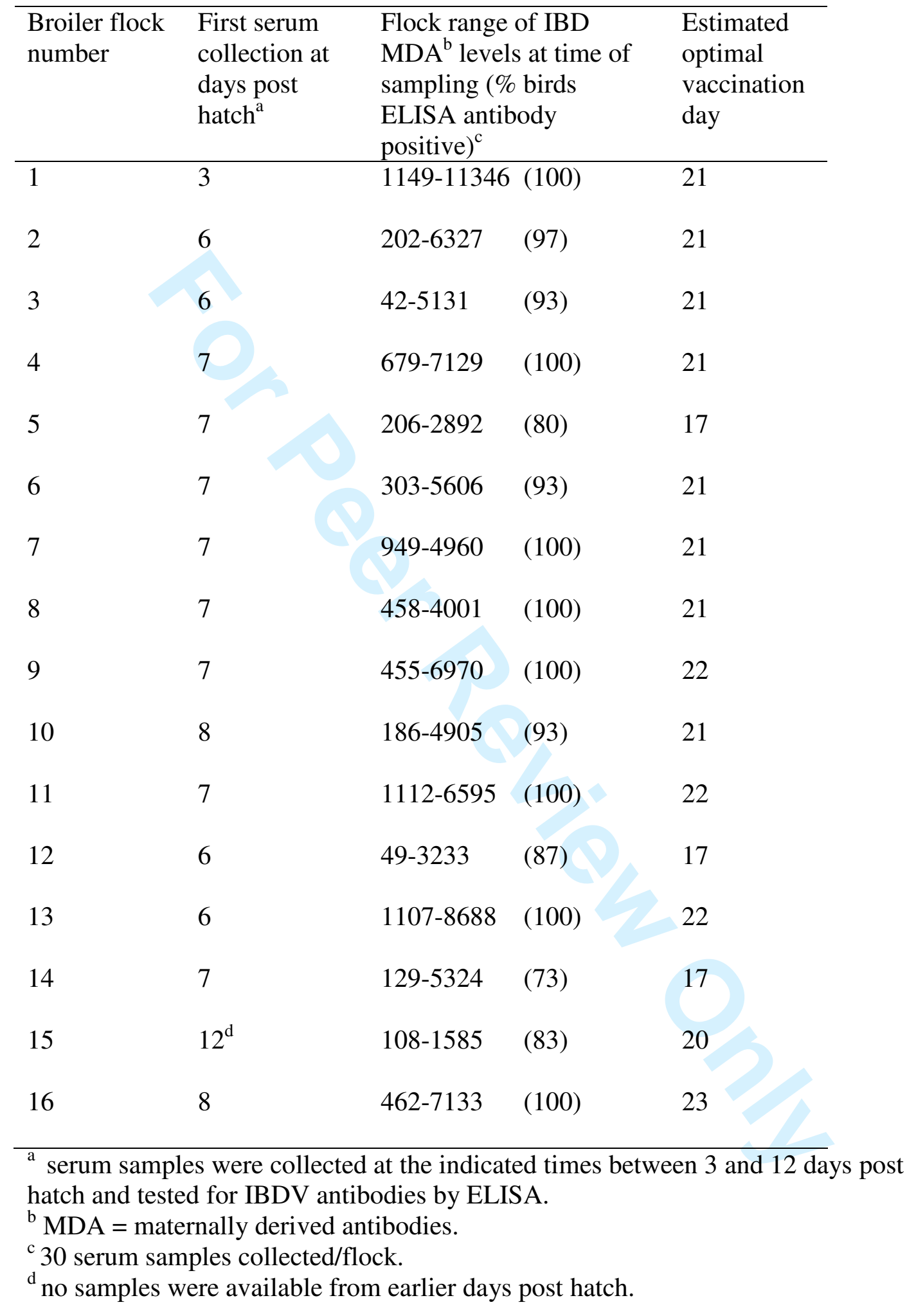


Table 2. Antibody decline between first and second serum sampling of flocks before the actual day of vaccination (Trial 1)

\begin{tabular}{llllll}
\hline $\begin{array}{l}\text { Broiler } \\
\text { flock } \\
\text { number }\end{array}$ & $\begin{array}{l}\text { Parent flock } \\
\text { vaccinated } \\
\text { live/inactivated } \\
\text { (age in weeks) }\end{array}$ & $\begin{array}{l}\text { Mean MDA }{ }^{\mathrm{a}} \text { ELISA antibody level } \\
\text { at age of first sampling }\end{array}$ & $\begin{array}{l}\text { Mean MDA ELISA antibody } \\
\text { level at age of second sampling }\end{array}$ \\
\hline 9 & & Day bled & ELISA titre \pm SD & Day bled & ELISA titre \pm SD \\
2 & $+/{ }^{\mathrm{c}}(28)$ & 7 & $4023 \pm 1721$ & 17 & $270 \pm 167$ \\
3 & $+/+(31)$ & 6 & $3194 \pm 1536$ & 18 & $959 \pm 598$ \\
11 & $+/+(31)$ & 6 & $2782 \pm 1421$ & 18 & $926 \pm 646$ \\
10 & $+/+(35)$ & 7 & $3690 \pm 1173$ & 17 & $413 \pm 219$ \\
1 & $+/+(37)$ & 8 & $2218 \pm 1356$ & 16 & $266 \pm 223$ \\
4 & $+/+(38)$ & 3 & $6143 \pm 1993$ & 17 & $592 \pm 434$ \\
6 & $+/+(52)$ & 7 & $2791 \pm 1332$ & 18 & $355 \pm 241$ \\
12 & $+/-{ }^{\mathrm{d}}(31)$ & 7 & $2128 \pm 1423$ & 17 & $228 \pm 170$ \\
13 & $+/-(38)$ & 6 & $1211 \pm 837$ & ND & ND \\
8 & $+/-(39)$ & 6 & $4441 \pm 1642$ & 17 & $199 \pm 131$ \\
14 & $+/-(41)$ & 7 & $2072 \pm 901$ & 18 & $448 \pm 466$ \\
7 & $+/-(44)$ & 7 & $1122 \pm 1260$ & ND & ND \\
5 & $+/-(52)$ & 7 & $2711 \pm 804$ & 18 & $256 \pm 181$ \\
15 & $+/-(53)$ & 7 & $1048 \pm 712$ & 15 & $146 \pm 142$ \\
16 & $+/-(54)$ & 12 & $794 \pm 385$ & 18 & $172 \pm 110$ \\
\hline
\end{tabular}

Serum samples were collected at the indicated times and tested for IBDV antibodies by ELISA. The second serum samples were collected 2 to 5 days before the estimated optimal vaccination day.

${ }^{a}$ MDA - maternally derived antibodies.

b30 samples/flock.

${ }^{c}+/+$ flock was vaccinated by drinking water with a live IBDV vaccine and boosted with an inactivated IBDV vaccine by injection.

${ }^{d}+$ - flock was vaccinated by drinking water with a live IBDV vaccine.

${ }^{\mathrm{e}} \mathrm{ND}$-no second serum samples were available from these flocks. 
Table 3. Retrospective determination of the optimal IBD vaccination time and grouping of the flocks based on actual vaccination before, at, or after the optimal time (Trial 2)

\begin{tabular}{|c|c|c|c|}
\hline $\begin{array}{l}\text { Assigned groups and } \\
\text { broiler flock } \\
\text { numbers }^{\mathrm{a}}\end{array}$ & $\begin{array}{l}\text { Estimated } \\
\text { optimal } \\
\text { vaccination } \\
\text { time in days } \\
\text { post hatch } \\
\end{array}$ & $\begin{array}{l}\text { Actual day of } \\
\text { vaccination ( } \% \text { birds } \\
\text { with MDA }{ }^{b} \text { titre above } \\
\text { the ELISA cut-off at day } \\
\text { of vaccination) }\end{array}$ & $\begin{array}{l}\text { Difference in } \\
\text { days between } \\
\text { optimal and } \\
\text { actual } \\
\text { vaccination time }\end{array}$ \\
\hline \multicolumn{4}{|c|}{ Group 1 vaccinated before the optimal day } \\
\hline 9 & 22 & $14(92)$ & -8 \\
\hline 3 & 24 & $21 \quad(54)$ & -3 \\
\hline 4 & 24 & $21 \quad(54)$ & -3 \\
\hline 12 & 22 & $21 \quad(50)$ & -1 \\
\hline \multicolumn{4}{|c|}{ Group 2 vaccinated at the optimal day } \\
\hline 1 & 18 & $18 \quad(54)$ & 0 \\
\hline 5 & 21 & 21 & 0 \\
\hline 6 & 19 & 19 (13) & 0 \\
\hline 8 & 20 & $20 \quad(21)$ & 0 \\
\hline 10 & 20 & $20 \quad(0)$ & 0 \\
\hline \multicolumn{4}{|c|}{ Group 3 vaccinated after the optimal day } \\
\hline 15 & 21 & $22(8)$ & +1 \\
\hline 18 & 21 & $23 \quad(17)$ & +2 \\
\hline 19 & 21 & $23 \quad(13)$ & +2 \\
\hline 2 & 18 & 21 & +3 \\
\hline 11 & 21 & $24 \quad(17)$ & +3 \\
\hline 7 & 14 & $18 \quad(0)$ & +4 \\
\hline 13 & 20 & $24 \quad(0)$ & +4 \\
\hline 16 & 18 & $22 \quad(0)$ & +4 \\
\hline 14 & 17 & $22 \quad(0)$ & +5 \\
\hline 17 & 17 & $23 \quad(13)$ & +6 \\
\hline 20 & 17 & $23 \quad(0)$ & +6 \\
\hline
\end{tabular}

a 30 serum samples per flock.

${ }^{\mathrm{b}} \mathrm{MDA}=$ maternally derived antibodies. 


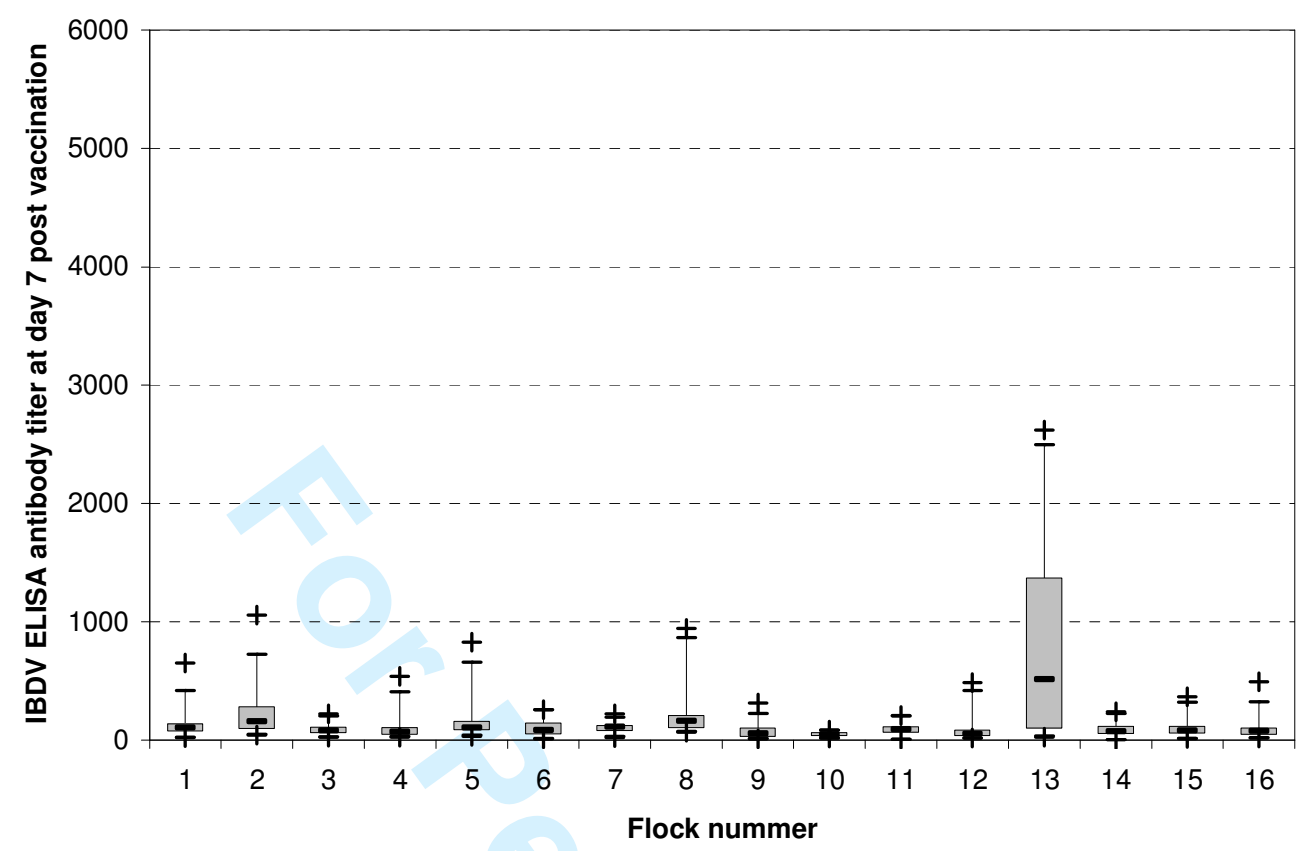

Figure 1a 


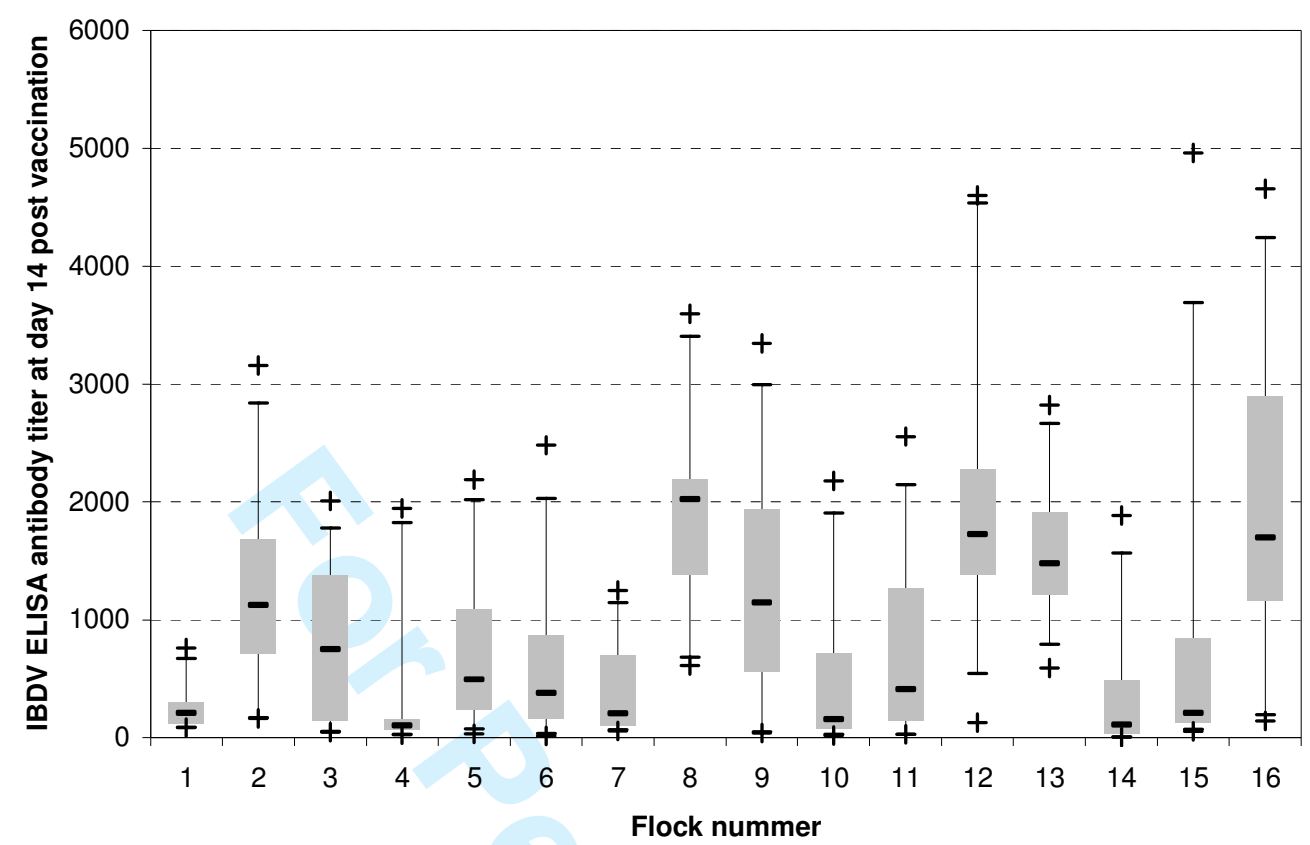

Figure 1b 




Figure 1c:

Figure 1: IBDV-antibody development after vaccination at the optimal time point (Trial 1).

Serum samples were collected at $7(a), 14(b)$, and 21 (c) days post vaccination and tested for IBDV antibodies by ELISA. $n=30 /$ flock. The ELISA data are presented in the box \& whisker diagram: the short line within the grey rectangular box represents the median of the variables; the upper and lower boarder of the rectangular box represent the 25 and 75 quartiles, respectively; the whiskers indicate highest and lowest value; outliers are indicated by $a+$. 


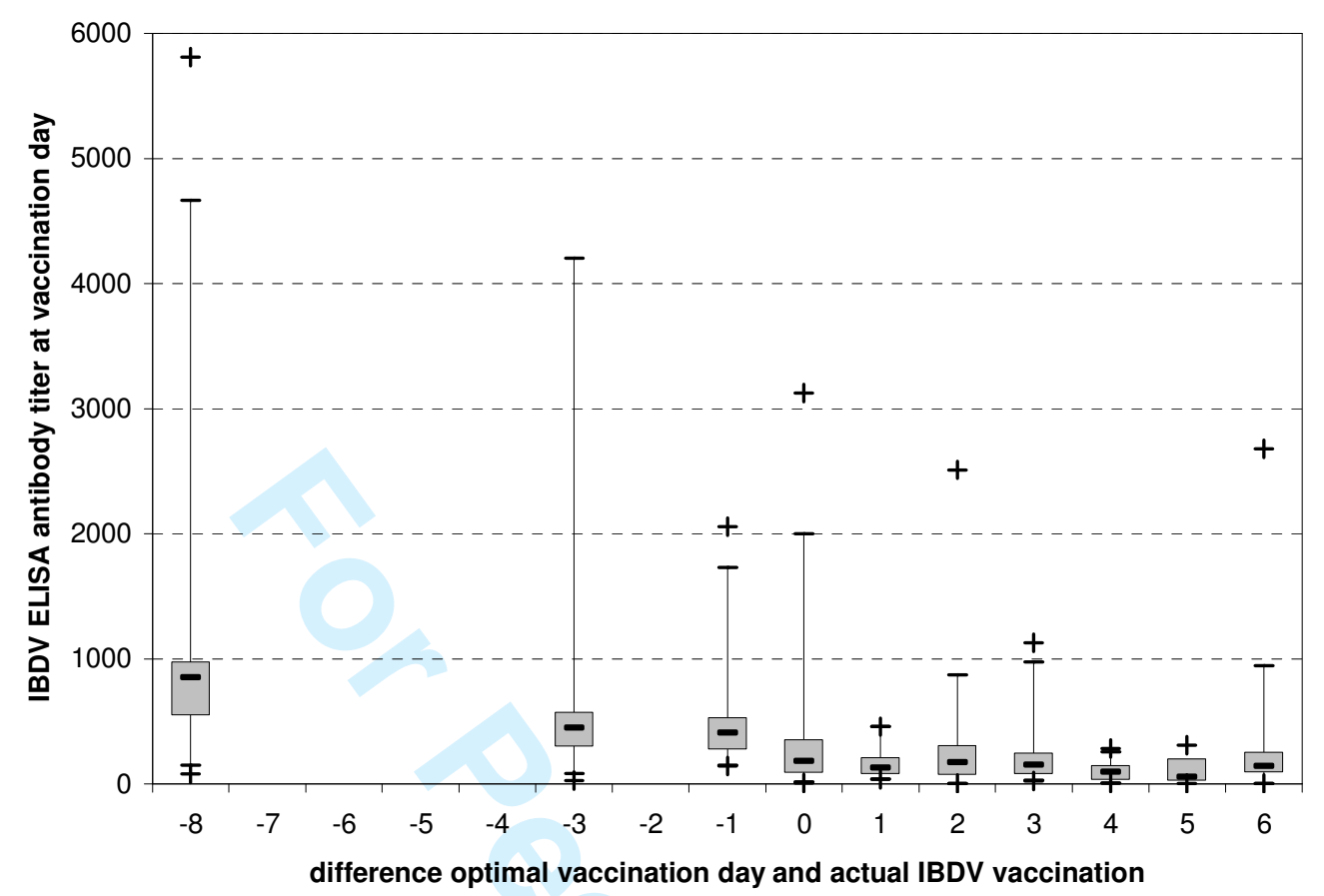

Figure 2a 


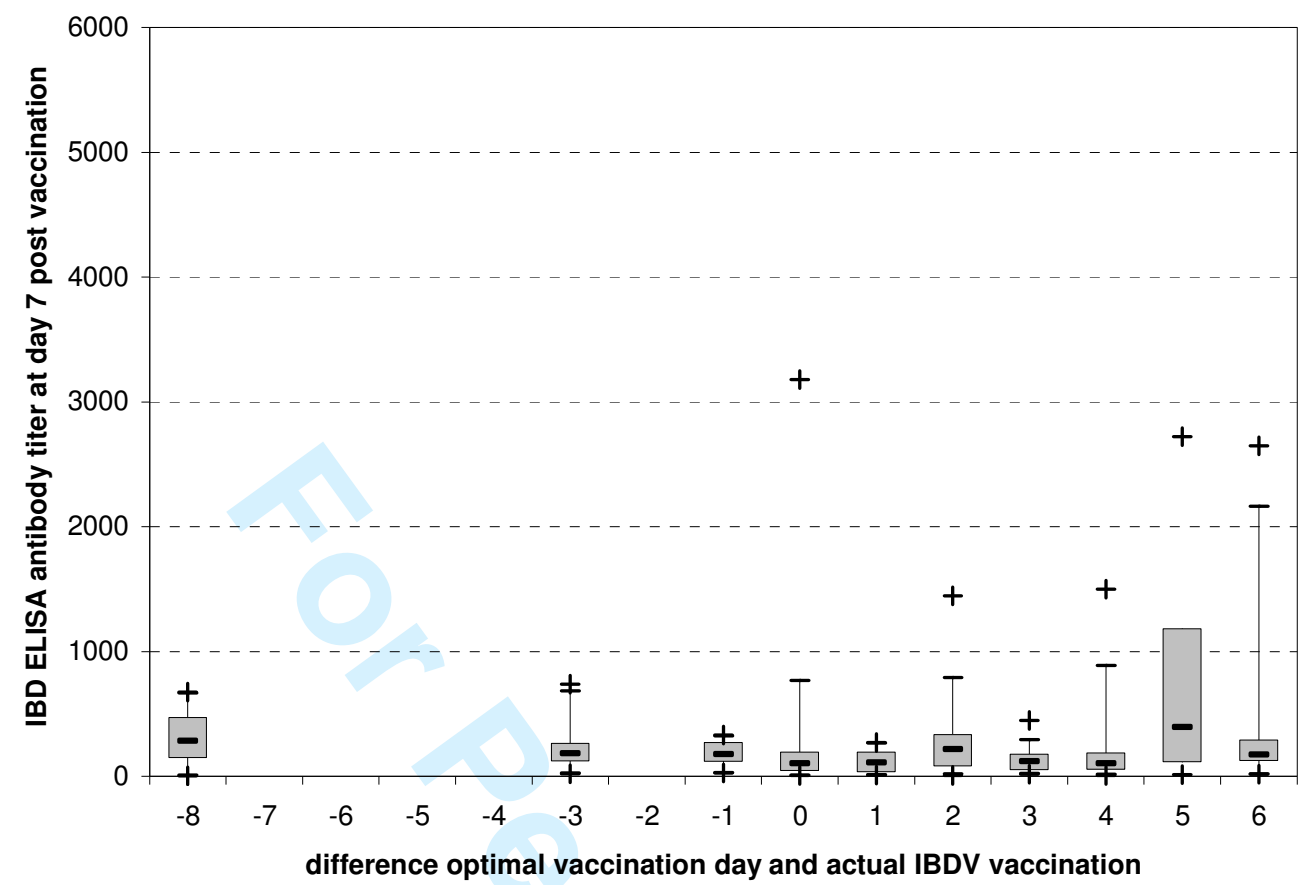

Figure $2 b$ 




Figure 2c 




Figure 2d

Figure 2: IBDV-antibody development after vaccination before (days -8, -3, -1), at (day 0), and after the optimal time point (days 1 to 6) (Trial 2). Serum samples were collected at the day of vaccination (a), $7(b), 14(c)$, and $21(d)$ days post vaccination and tested for IBDV antibodies by ELISA; at 21 days post vaccination, flocks vaccinated at day 2 and 6 after the optimal time had been slaughtered, no data are available for these flocks at this day point. 30 serum samples/flock; the number of flocks per day point varied: day $-8,-1,+1,+5: n=1$; day $-3,+2,+3,+6: n=2 ;$ day $+4: n=3 ;$ day $0: n=5$. The ELISA data are presented in the box \& whisker diagram: the short line within the grey rectangular box represents the median of the variables; the upper and lower boarder of the rectangular box represent the 25 and 75 quartiles, respectively; the whiskers indicate highest and lowest value; outliers are indicated by $a+$ 




Figure 3. Development of histological bursa lesions and detection of IBDV by RT-PCR after vaccination of broiler flocks with an intermediate IBDV strain (Trial 1). Flocks 3, 8, 9, and 16 were slaughtered before 21 days post vaccination, and no data were available for this time point. $d p v=$ days post IBDV vaccination. $n=5$ bursae per flock were investigated. $+=$ detection of IBDV by RT-PCR from pooled bursa tissue $(n=5 / p o o l) ;-=$ no detection of IBDV by RT-PCR. IBDV detection in flocks 5, 8, 9 was possible after propagation of IBDV in embryonated chicken eggs and consecutive RT-PCR. 